\title{
Equilíbrio, Coordenação e Agilidade de Idosos Submetidos à Prática de Exercícios Físicos Resistidos
}

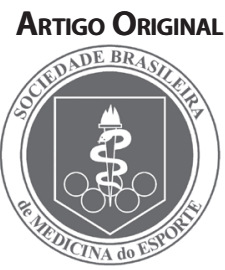

\section{Balance, Coordination and Agility of Older Individuals Submitted to Physical Resisted Exercises Practice}

\begin{abstract}
Andressa da Silva ${ }^{1,2,3}$
Gustavo JM Almeida ${ }^{1,2}$

Ricardo C. Cassilhas ${ }^{1,3}$

Moises Cohen 2,4

Maria Stella Peccin 1,6

Sergio Tufik $1,3,5,7$

Marco Túlio de Mello 1,3,5,8

1. Centro de Estudos em

Psicobiologia e Exercício (CEPE)

2. Universidade São Marcos, Brasil

3. Departamento de Psicobiologia,

Universidade Federal de São Paulo

(UNIFESP), Brasil

4. Departamento de Ortopedia e

Traumatologia, Universidade Federa

de São Paulo (UNIFESP), Brasil

5. Pesquisadores do CNPq

6. Professora Adjunta I do Campus

de Santos da Universidade Federal

de São Paulo (UNIFESP)

7. Presidente da AFIP

8. Professor Adjunto III do

Departamento de Psicobiologia da

Universidade Federal de São Paulo

(UNIFESP)
\end{abstract}

\section{Endereço para correspondência:}

Marco Túlio de Mello

Rua Marselhesa 535 - Vila Clementino

São Paulo - SP - Brasil - CEP 04020-060

Telefones: +55 1155720177

+551150836900

Email: tmello@psicobio.epm.br

Submetido em 27/09/2006

Versão final recebida em 08/03/2007

Aceito em 29/03/2007

\begin{abstract}
RESUMO
Objetivos: Avaliar o equilíbrio, a coordenação e a agilidade dos idosos submetidos a exercícios físicos. MÉTODOS: 61 idosos do gênero masculino, com idades entre os 60-75 anos, designados aleatoriamente para um grupo de exercícios resistidos com carga progressiva $(n=39)$ ou para um controle submetido a exercícios sem carga ( $n=22)$. O grupo exercício resistido participou de um programa de 24 semanas, com 3 visitas por semana, em dias não consecutivos. Foram avaliados após o término do treinamento, pela Escala de Equilíbrio de Berg, do Teste de Tinetti e do Timed UP \& GO. Resultados: Comparando-se os dois grupos verificou-se um melhor desempenho estatisticamente significativo para o grupo experimental em relação ao controle para os testes Timed "Up \& Go". ( $p=0,02)$ e para o Tinetti Total $(p=0.046)$ e para o Tinetti marcha ( $p=0.029)$. Desta forma, não encontramos diferença na Escala de Equilíbrio de Berg e no teste de Tinetti equilíbrio. Conclusão: O programa de treinamento de força durante 24 semanas mostrou-se favorável na melhora dos desempenhos funcional e motores de idosos.
\end{abstract}

Palavras-chave: equilíbrio, quedas, idosos, investigação clínica, exercício físico.

\begin{abstract}
Objectives: To evaluate balance, coordination and agility of older subjects submitted to physical exercise. Methods: 61 male older subjects, aged between 60-75 years, randomly assigned to a resisted exercises with gradual load $(n=39)$ group or to a control group submitted to exercise without load $(n=22)$. The resisted exercise group participated in a 24 week-program, with 3 weekly visits, in not consecutive days. After the training ending, they were evaluated through the Berg Balance Scale and the Tinetti and Timed UP \& GO tests. Results: Comparing the two groups, better statistical significant performance was verified for the experimental group for the Timed "Up \& Go" ( $p=0.02)$, for the Total Tinetti ( $p=0.046)$ and for the Tinetti gait tests $(p=0.029)$. Therefore, we did not find difference in the Berg Balance Scale or in the Tinetti balance test. Conclusion: The strength training program during 24 weeks behaved favorable in improving functional and motor performance of older subjects.
\end{abstract}

Keywords: balance, falls, the elderly, clinical inquiry, physical exercise.

\section{INTRODUÇÃO}

O equilíbrio é um processo complexo que depende da integração da visão, da sensação vestibular e periférica, dos comandos centrais e respostas neuromusculares e, particularmente, da força muscular e do tempo de reação. Um declínio da função relacionado à idade pode ser demonstrado em todas as partes desses sistemas tendo como resultado o fato de que um terço da população acima de 65 anos sofrem quedas a cada ano(1).

Para obter um melhor equilíbrio, um indivíduo procura manter o seu centro de massa corporal dentro dos seus limites de estabilidade, sendo determinada pela habilidade em controlar a postura sem alterar a base de suporte ${ }^{(1)}$.

No Brasil, 30\% dos idosos caem pelo menos uma vez por ano e quanto maior a idade maior a chance de queda, sendo que 32\% estão entre os 65 e os 74 anos, 35\% entre os 75 e os 84 anos e $51 \%$ acima dos 85 anos. Estas quedas ocorrem mais nas mulheres do que nos homens da mesma faixa etária. Os idosos dos 75 aos 84 anos que necessitam de ajuda para as atividades da sua vida diária (comer, tomar banho, higiene íntima, vestir-se, sair da cama, incontinência urinária e fecal) têm uma probabilidade de cair 14 vezes mais do que pessoas da mesma idade que são independentes. De todas as quedas, $5 \%$ resultam em fraturas e 5 a 10\% em ferimentos importantes que necessitam de cuidados médicos ${ }^{(2)}$.

A escala de equilíbrio de Berg, criada em 1992 por Katherine Berg, tem tido ampla utilização para avaliar o equilíbrio nos indivíduos da terceira idade acima dos 60 anos ${ }^{(3,4)}$. Esta escala foi traduzida e adaptada para a língua portuguesa por Miyamoto et al. ${ }^{(3)}$, na sua dissertação de mestrado, pelo que a versão brasileira é um instrumento confiável para ser usado na avaliação do equilíbrio dos pacientes idosos.

A EEB é uma avaliação funcional do desempenho do equilíbrio, baseada em 14 itens comuns do dia a dia que avaliam o controle postural, incluindo o estável e o antecipatório ${ }^{(5)}$ e que requerem diferentes forças, equilíbrio dinâmico e flexibilidade(6). 
Berg et al. ${ }^{(5)}$ relatou que o teste tem uma boa objetividade de teste-reteste $(I C C=0,98)$ e uma boa consistência interna (Alpha de Cronbach=096), conseguindo diferenciar os idosos mais propensos a quedas, além de estar correlacionado a outros testes de equilíbrio e de mobilidade, incluindo o teste de Tinetti, o Timed Up and $G^{(7)}$.

Desta maneira, estudos recentes têm sido realizados na busca de novas informações sobre o efeito do exercício físico no equilíbrio dos idosos. Um estudo recente realizado por Kenneth e Behm ${ }^{(8)}$ descreveu que, o efeito do exercício de resistência no tamanho e na força do músculo, tem sido claramente documentado, embora evidências sugiram que o treino de resistência, que é um treino de equilíbrio ausente, também tem um efeito positivo no equilíbrio. Foi encontrado que os exercícios de força contribuem para um melhor equilíbrio e marcha nas mulheres com idade superior ou igual a 57 anos, de forma que não foram usados os mesmos instrumentos de avaliação que utilizamos neste estudo.

Kenneth e Behm ${ }^{(8)}$, encontraram na sua pesquisa que a estabilidade da marcha melhorou significativamente mais no grupo de exercício resistido que no controle. Estes resultados mostraram que o mesmo ganho moderado de força $(17,6 \%)$ pode beneficiar a marcha e o equilíbrio, fornecendo assim uma base sólida para o incentivo do treinamento de força de baixa intensidade para os indivíduos com limitações funcionais.

Recentemente, Kenneth e Behm ${ }^{(8)}$, testaram se um programa de treinamento pode restabelecer o equilíbrio nos indivíduos velhos. 0 efeito do treino de força e de resistência no equilíbrio nos idosos (idade entre os 65 e os 85 anos), com equilíbrio reduzido, mostrou que tempo curto de treinamento de força e de resistência não tem efeito restaurador no equilíbrio.

Sendo assim, diversos autores vendo a necessidade de prevenir quedas criaram e testaram instrumentos que quantificam o equilíbrio e a coordenação como, por exemplo, a escala de equilíbrio de Berg e Timed Up and Go entre outros ${ }^{(9)}$ que foram utilizados como critérios de avaliação no presente estudo.

\section{OBJETIVO}

Avaliar o equilíbrio, a coordenação e a agilidade dos idosos submetidos a exercícios físicos.

\section{MÉTODOS}

Foram avaliados 61 "idosos bem sucedidos", com idades entre os 60 e os 75 anos, que participaram voluntariamente do estudo, tendo sido designados aleatoriamente por meio de um programa de computador para o grupo experimental (exercícios físicos resistidos com carga progressiva) ( $n=39)$ ou para o controle submetido a exercícios resistidos sem carga $(n=22)$.

O projeto foi aprovado pelo Comitê de Ética em Pesquisa da Unifesp (0029/06). O estudo foi realizado no Centro de Estudos em Psicobiologia e Exercício (CEPE), no período da manhã e com marcação prévia.

Os voluntários foram divididos em dois grupos: controle e experimental (exercícios) a 80\% de 1RM.

\section{PROTOCOLO DE TREINAMENTO}

\section{Grupo Experimental}

O treinamento para o grupo experimental consistiu de 3 sessões por semana em dias alternados, com a duração de uma hora cada sessão, durante 6 meses, completando 72 sessões de treinamento. No início de cada sessão, os grupos experimentais foram submetidos a um breve aquecimento de 10 minutos, seguindo-se exercícios de alongamento. A carga correspondente ao treinamento para o grupo exercício foi de $80 \%$ de 1RM, tendo sido utilizado o modelo alternado por seguimento com 2 séries de 8 repetições para cada uma, com intervalos de descanso de 1 minuto e 30 segundos entre elas e de 3 minutos entre cada aparelho.

\section{Grupo Controle}

O grupo controle não foi submetido ao treinamento com sobrecarga ao longo do período da intervenção. Contudo, este grupo freqüentou o CEPE uma vez por semana limitando-se a praticar, sem sobrecarga, aquecimento e alongamento, o mesmo protocolo de exercício dos grupos experimentais (6 exercícios em modelo alternado por seguimento com 2 séries de 8 repetições, com os mesmos intervalos de descanso). Este modelo de grupo controle se justificou pelo fato de que se pode eliminar o viés da aprendizagem neuromotora e os fatores de interação social, que por ventura possam mascarar o real efeito do treinamento resistido nas variáveis cognitivas estudadas no grupo exercício em um estudo paralelo.

Os exercícios realizados em ambos os grupos (experimental e controle) foram: "Leg Press", membro inferior (quadríceps femoral); "Leg Curl", membro inferior (especialmente os isquiotibiais); "Vertical Traction", tronco e membro superior (especialmente o grande dorsal e secundariamente os bíceps); "Chest Press", tronco e membro superior (especialmente o peitoral maior e secundariamente os tríceps); "Abdominal Crunch", abdominal; "Lower back", musculatura paravertebral.

As avaliações de equilíbrio foram realizadas após completar as 72 sessões de treinamento. Os testes foram realizados em um período em que não houve outro tipo de treinamento em avaliação, para evitar qualquer tipo de interferência no processo avaliativo. Os testes realizados foram os indicados em seguida.

\section{Escala de Equilíbrio de Berg}

A escala de equilíbrio de Berg tem uma pontuação máxima de 56 que pode ser alcançada, possuindo cada item uma escala ordinal de 5 alternativas que variam de 0 a 4 pontos. 0 teste é simples, fácil de administrar e seguro para a avaliação de pacientes idosos. Ele somente requer um cronômetro e uma régua como equipamentos e a sua execução leva-se em torno de 15 minutos $^{(6)}$. (Tabela 1)

\section{Teste de Tinetti}

O Teste de Tinetti tem sido usado para avaliar o equilíbrio e as anormalidades da marcha. $O$ teste consiste de 16 itens, em que 9 são para o equilíbrio do corpo (TABELA 2.1) e 7 para a marcha (TABELA 2.2). O Teste de Tinetti classifica os aspectos da marcha como a velocidade, a distancia do passo, a simetria e o equilíbrio em pé, o girar e também as mudanças com os olhos fechados. A contagem para cada exercício varia de 0 a 1 ou de 0 a 2, com uma contagem mais baixa que indica uma habilidade física mais pobre. A pontuação total é a soma da pontuação do equilíbrio do corpo e a da marcha. A pontuação máxima é de 12 pontos para a marcha, de 16 para o equilíbrio do corpo e de 28 para a total ${ }^{(10)}$.

\section{Teste Timed Up and Go}

Os pacientes ficam sentados em uma cadeira normal $(45 \mathrm{~cm}$ de altura) com sua parte traseira de encostada à cadeira. Foram instruídos

\footnotetext{
"Idoso bem-sucedido" é aquele que apresenta características de sabedoria, persistência, rapidez e criatividade; que se mantém ativo, esperançoso e produtivo; e que é generoso, construtivo, cordial, bem-humorado, agradável e interessado pelas pessoas.
} 
1) Posição sentada para posição em pé para se apoiar.

\section{2) Permanecer em pé sem apoio}

Instrução: Por favor, fique em pé por 2 minutos sem se apoiar

Se o paciente for capaz de permanecer em pé por 2 minutos sem apoio, dê o número total de pontos o item $N^{\circ} 3$.

3) Permanecer sentado sem apoio nas costas, mas com os pés apoiados no chão ou num banquinho Instrução: Por favor, fique sentado sem apoiar as costas com os braços cruzados por 2 minutos.

4) Posição em pé para posição sentada Instrução: Por favor, sente-se. Instrução: Por favor, levante-se. Tente não usar suas mãos Continue com o item $\mathrm{N}^{\circ} 4$.

(4) capaz de levantar-se sem utilizar as mãos e estabilizar-se independentemente

(3) capaz de levantar-se independentemente utilizando as mãos

(2) capaz de levantar-se utilizando as mãos após diversas tentativas

(1) necessita de ajuda mínima para levantar-se ou estabilizar-se

(0) necessita de ajuda moderada ou máxima para levantar-se

(4) capaz de permanecer em pé com segurança por 2 minutos

(3) capaz de permanecer em pé por 2 minutos com supervisão

(2) capaz de permanecer em pé por 30 segundos sem apoio

(1) necessita de várias tentativas para permanecer em pé por 30 segundos sem apoio

(0) incapaz de permanecer em pé por 30 segundos sem apoio

(4) capaz de permanecer sentado com segurança e com firmeza por 2 minutos

(3) capaz de permanecer sentado por 2 minutos sob supervisão

(2) capaz de permanecer sentado por 30 segundos

(1) capaz de permanecer sentado por 10 segundos

(0) incapaz de permanecer sentado sem apoio durante 10 segundos

(4) senta-se com segurança com uso mínimo das mãos

(3) controla a descida utilizando as mãos

(2) utiliza a parte posterior das pernas contra a cadeira para controlar a descida

(1) senta-se independentemente, mas tem descida sem controle

(0) necessita de ajuda para sentar-se

\section{5) Transferências}

Instrução: Arrume as cadeiras perpendicularmente ou uma de frente para a outra para uma transferência em pivô. Peça ao paciente para transferir-se de uma cadeira com apoio de braço para uma cadeira sem apoio de braço, e vice-versa.

6) Permanecer em pé sem apoio com os olhos fechados Instrução: Por favor, fique em pé e feche os olhos por 10 segundos.

(4) capaz de transferir-se com segurança com uso mínimo das mãos

(3) capaz de transferir-se com segurança com o uso das mãos

(2) capaz de transferir-se seguindo orientações verbais e/ou supervisão

(1) necessita de uma pessoa para ajudar

(0) necessita de duas pessoas para ajudar ou supervisionar para realizar a tarefa com segurança

(4) capaz de permanecer em pé por 10 segundos com segurança

(3) capaz de permanecer em pé por 10 segundos com supervisão

(2) capaz de permanecer em pé por 3 segundos

(1) incapaz de permanecer com os olhos fechados durante 3 segundos, mas mantém-se em pé

(0) necessita de ajuda para não cair

7) Permanecer em pé sem apoio com os pés juntos Instrução: Junte seus pés e fique em pé sem se apoiar.

(4) capaz de posicionar os pés juntos independentemente e permanecer por 1 minuto com segurança

(3) capaz de posicionar os pés juntos independentemente e permanecer por 1 minuto com supervisão

(2) capaz de posicionar os pés juntos independentemente e permanecer por 30 segundos

(1) necessita de ajuda para posicionar-se, mas é capaz de permanecer com os pés juntos durante 15 segundos

(0) necessita de ajuda para posicionar-se e é incapaz de permanecer nessa posição por 15 segundos

8) Alcançar a frente com o braço estendido permanecendo em pé

Instrução: Levante o braço a $90^{\circ}$. Estique os dedos e tente alcançar a frente o mais longe possível.

10) Virar-se e olhar para trás por cima dos ombros direito e esquerdo enquanto permanece em pé

Instrução: Vire-se para olhar diretamente atrás de você por cima do seu ombro esquerdo sem tirar os pés do chão. Faça o mesmo por cima do ombro direito.

\section{1) Girar 360 graus}

Instrução: Gire-se completamente ao redor de si mesmo. Pausa. Gire-se completamente ao redor de si mesmo em sentido contrário.

12) Posicionar os pés alternadamente no degrau ou banquinho enquanto permanece em pé sem apoio Instrução: Toque cada pé alternadamente no degrau/banquinho. Continue até que cada pé tenha tocado o degrau/ banquinho quatro vezes.

13) Permanecer em pé sem apoio com um pé à frente Instrução: Coloque um pé diretamente à frente do outro na mesma linha, se você achar que não irá conseguir, coloque o pé um pouco mais à frente do outro pé e levemente para o lado.

14) Permanecer em pé sobre uma perna Instrução: Fique em pé sobre uma perna o máximo que você puder sem se segurar.
(4) pode avançar a frente $>25 \mathrm{~cm}$ com segurança

(3) pode avançar a frente $>12,5 \mathrm{~cm}$ com segurança

(2) pode avançar a frente $>5 \mathrm{~cm}$ com segurança

(1) pode avançar a frente, mas necessita de supervisão

(0) perde o equilíbrio na tentativa, ou necessita de apoio externo

(4) olha para trás de ambos os lados com uma boa distribuição do peso

(3) olha para trás somente de um lado, o lado contrário demonstra menor distribuição do peso

(2) vira somente para os lados, mas mantém o equilíbrio

(1) necessita de supervisão para virar

(0) necessita de ajuda para não perder o equilíbrio ou cair

\section{(4) capaz de girar 360 graus com segurança em 4 segundos ou menos}

(3) capaz de girar 360 graus com segurança somente para um lado em 4 segundos ou menos

(2) capaz de girar 360 graus com segurança, mas lentamente

(1) necessita de supervisão próxima ou orientações verbais

(0) necessita de ajuda enquanto gira

(4) capaz de permanecer em pé independentemente e com segurança, completando 8 movimentos em 20 segundos

(3) capaz de permanecer em pé independentemente e completar 8 movimentos em $>20$ segundos

(2) capaz de completar 4 movimentos sem ajuda

(1) capaz de completar >2 movimentos com o mínimo de ajuda

(4) capaz de colocar um pé imediatamente à frente do outro, independentemente, e permanecer por 30 segundos

(3) capaz de colocar um pé um pouco mais à frente do outro e levemente para o lado, independentemente, e permanecer por 30 segundos

(2) capaz de dar um pequeno passo, independentemente, e permanecer por 30 segundos

(1) necessita de ajuda para dar o passo, porém permanece por 15 segundos

(0) perde o equilíbrio ao tentar dar um passo ou ficar de pé

(4) capaz de levantar uma perna independentemente e permanecer por $>10$ segundos

(3) capaz de levantar uma perna independentemente e permanecer por 5-10 segundos

(2) capaz de levantar uma perna independentemente e permanecer por $\geq 3$ segundos

(1) tenta levantar uma perna, mas é incapaz de permanecer por 3 segundos, embora permaneça em pé independentemente

(0) incapaz de tentar, ou necessita de ajuda para não cair
(0) incapaz de tentar, ou necessita de ajuda para não cair 
Tabela 2. Teste de Tinetti ${ }^{(10)}$

\subsection{Teste de Equilíbrio}

(Instruções: Sujeito sentado em uma cadeira rígida, sem braços)

\begin{tabular}{|c|c|}
\hline 1) Equilíbrio sentado: & $\begin{array}{l}\text { (0) Inclina-se ou desliza na cadeira } \\
\text { (1) Estável, seguro }\end{array}$ \\
\hline 2) Levanta-se da cadeira: & $\begin{array}{l}\text { (0) Incapaz sem ajuda } \\
\text { (1) Capaz, usa membros superiores para auxiliar } \\
\text { (2) Capaz sem usar membros superiores }\end{array}$ \\
\hline 3) Tentativas para se levantar: & $\begin{array}{l}\text { (0) Incapaz sem ajuda } \\
\text { (1) Capaz, requer mais de uma tentativa } \\
\text { (2) Capaz de se levantar, uma tentativa }\end{array}$ \\
\hline $\begin{array}{l}\text { 4) Equilíbrio de pé imediato } \\
\text { (primeiros } 5 \text { segundos) }\end{array}$ & $\begin{array}{l}\text { (0) Instável (cambaleia, move os pés, oscila o tronco) } \\
\text { (1) Estável, mas usa dispositivo de auxílio à marcha } \\
\text { (2) Estável sem dispositivo de auxílio }\end{array}$ \\
\hline 5) Equilíbrio de pé: & $\begin{array}{l}\text { (0) Instável } \\
\text { (1) Instável, mas aumenta a base de suporte (entre os } \\
\text { calcanhares }>10 \mathrm{~cm} \text { de afastamento) e usa dispositivo } \\
\text { de auxílio } \\
\text { (2) Diminuição da base sem dispositivo de auxílio }\end{array}$ \\
\hline $\begin{array}{l}\text { 6) Desequilíbrio no esterno } \\
\text { (sujeito na posição de pé com } \\
\text { os pés o mais próximo pos- } \\
\text { sível, o examinador empurra } \\
\text { suavemente o sujeito na altu- } \\
\text { ra do esterno com a palma da } \\
\text { mão } 3 \text { vezes seguidas: }\end{array}$ & $\begin{array}{l}\text { (0) Começa a cair } \\
\text { (1) Cambelaia, se agarra e se segura em si mesmo } \\
\text { (2) Estável }\end{array}$ \\
\hline 7) Olhos fechados: & $\begin{array}{l}\text { (0) Instável } \\
\text { (1) Estável }\end{array}$ \\
\hline 8) Girar $360^{\circ}$ : & $\begin{array}{l}\text { (0) Instabilidade (se agarra, cambaleia) } \\
\text { (1) Passos descontinuados } \\
\text { (2) Continuidade }\end{array}$ \\
\hline 9) Sentar-se: & $\begin{array}{l}\text { (0) Inseguro (não avalia bem a distância, cai na ca- } \\
\text { deira) } \\
\text { (1) Usa os braços ou não tem movimentos suaves } \\
\text { (2) Seguro, movimentos suave }\end{array}$ \\
\hline Escore de equilíbrio: & /16 \\
\hline
\end{tabular}

a ficar em pé; andar tão rapidamente quanto possível e com segurança por 3 m em uma linha reta no chão; retornar para a cadeira, sentandose na posição inicial(10).

\section{Análise Estatística}

A comparação entre os valores médios dos resultados dos dois grupos foi realizada pela análise de variância de duas vias. As comparações das avaliações aplicadas entre os grupos e as suas correlações foram realizadas por intermédio do teste de Mann-Whitney, sendo considerado como o valor de significância estatística p<0,05.

\section{RESULTADOS}

Os resultados apresentados demonstraram as avaliações referentes à Escala de Equilíbrio de Berg, ao Teste de Tinetti e ao Timed UP \& GO.

No Gráfico 1 podemos perceber que não houve diferenças estatísticas significativas entre os grupos para a Escala de Equilíbrio de Berg $(p=0,571)$.

Para os dados apresentados para o Teste Timed UP \& GO (Gráfico 2) verificou-se uma diferença estatisticamente significativa para o grupo experimental $(7,95 \pm 1,31)$ em relação ao controle $(8,58 \pm 1,03)$ tendo valor de $\mathrm{p}=0,02^{*}$

Houve uma diferença estatisticamente significativa entre os gru-

\subsection{Teste de Marcha}

(Instruções: Sujeito de pé com o examinador, caminha num corredor ou na sala, primeiro no seu ritmo usual e, em seguida, rápido, porém muito seguro, com os dispositivos de auxílio à marcha usuais):

\begin{tabular}{|c|c|}
\hline $\begin{array}{l}\text { 1) Iniciação da } \\
\text { marcha: }\end{array}$ & $\begin{array}{l}\text { (0) Imediato e após o comando Vá (qualquer hesitação ou } \\
\text { múltiplas tentativas para iniciar) } \\
\text { (1) Sem hesitação }\end{array}$ \\
\hline \multirow[t]{2}{*}{$\begin{array}{l}\text { 2) Comprimento e } \\
\text { altura do passo: }\end{array}$} & $\begin{array}{l}\text { a) Perna D em balanceio: } \\
\text { (0) Não passa o membro E } \\
\text { (1) Passa o membro E } \\
\text { (0) Pé D não se afasta completamente do solo com o passo } \\
\text { (1) Pé D se afasta completamente do solo }\end{array}$ \\
\hline & $\begin{array}{l}\text { b) Perna E em balanceio } \\
\text { (0) Não passa o membro D } \\
\text { (1) Passa o membro D } \\
\text { (0) Pé E não se afasta completamente do solo com o passo } \\
\text { (1) Pé E se afasta completamente do solo }\end{array}$ \\
\hline 3) Simetria do passo: & $\begin{array}{l}\text { (0) Passos D e E desiguais } \\
\text { (1) Passos D e E parecem iguais }\end{array}$ \\
\hline $\begin{array}{l}\text { 4) Continuidade do } \\
\text { passo: }\end{array}$ & $\begin{array}{l}\text { (0) Parada ou descontinuidade entre os passos } \\
\text { (1) Passos parecem contínuos }\end{array}$ \\
\hline $\begin{array}{l}\text { 5) Desvio da linha reta } \\
\text { (distância aproximada } \\
\text { de } 3 \mathrm{~m} \times 30 \mathrm{~cm} \text { ): }\end{array}$ & $\begin{array}{l}\text { (0) Desvio marcado } \\
\text { (1) Desvio leve e moderado ou usa dispositivo de auxílio à } \\
\text { marcha } \\
\text { (2) Caminha em linha reta sem dispositivo de auxílio }\end{array}$ \\
\hline 6) Tronco: & $\begin{array}{l}\text { (0) Oscilação marcada ou usa dispositivo de auxílio à marcha } \\
\text { (1) Sem oscilação, mas com flexão de joelhos ou dor lombar } \\
\text { ou afasta os braços enquanto anda } \\
\text { (2) Sem oscilação, sem flexão, sem uso dos braços ou de dis- } \\
\text { positivo de auxílio à marcha }\end{array}$ \\
\hline 7) Base de apoio: & $\begin{array}{l}\text { (0) Calcanhares afastados } \\
\text { (1) Calcanhares quase se tocando durante a marcha }\end{array}$ \\
\hline Escore de marcha: & $/ 12$ \\
\hline
\end{tabular}

Escore Total ___ $/ 28$

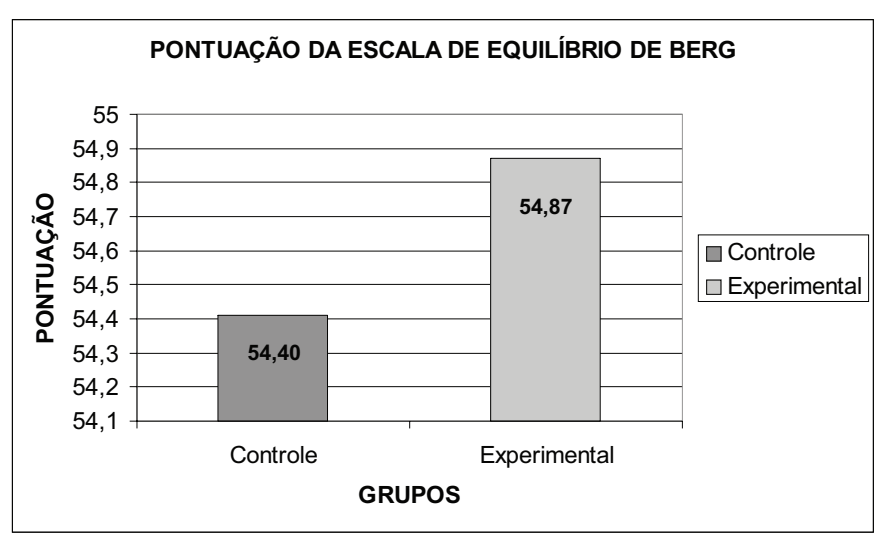

Gráfico 1. Comparação entre os Grupos para a Escala de Equilíbrio de Berg.

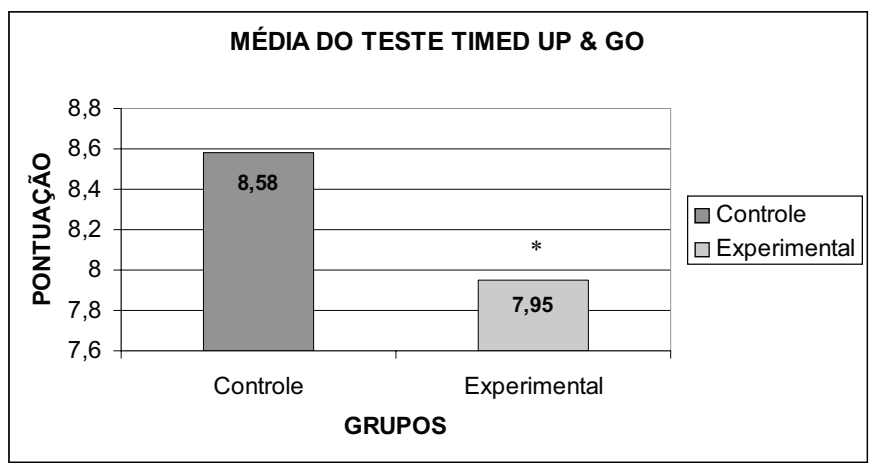

Gráfico 2. Comparação entre os Grupos para o Teste Timed UP \& GO,

*Nível de significância para $p<0,05$. 
pos experimental e o controle para o valor total do Teste de Tinetti $\left(p=0,046^{*}\right)$ e também para a marcha $\left(p=0,029^{*}\right)$, em que o experimental obteve melhor pontuação (Gráfico 3). Para o equilíbrio não encontramos significância $(p=0,331)$.

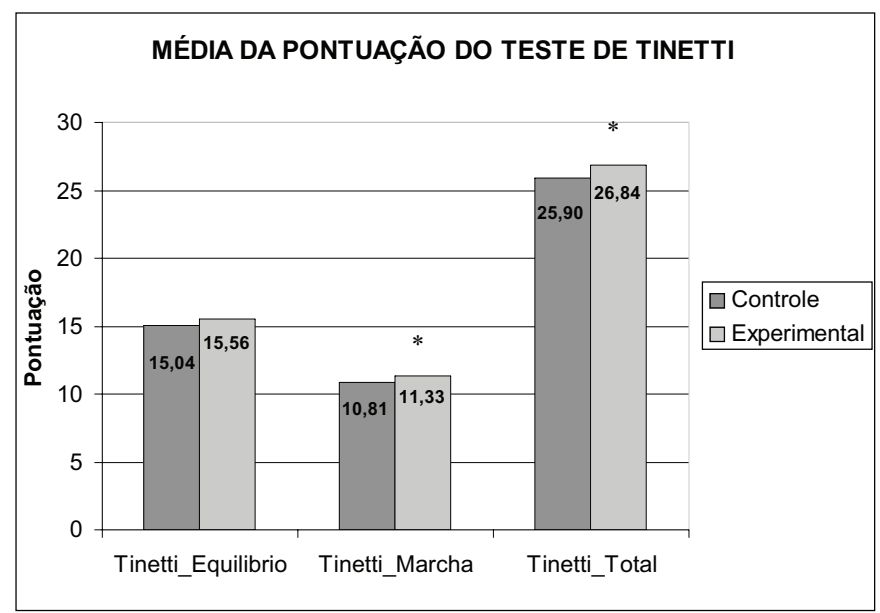

Gráfico 3. Comparação entre os Grupos para o Teste de Tinetti.

*Nível de significância para $p<0,05$.

A partir destes resultados o exercício resistido mostrou que, em todas as avaliações realizadas, houve um melhor desempenho para o grupo experimental em relação ao controle.

Ao realizar-se a correlação entre o BERG e o Timed "Up \& Go" encontrou-se uma correlação negativa $(r=-0,301 ; p=0,005)$ em que, quanto maior um (BERG) menor o outro (TUG).

Constatou-se também uma correlação negativa entre o equilíbrio $(r=-0,335)$ pelo teste de Tinetti e o seu valor total $(r=-0,385)$ com o Timed "Up \& $G o^{\prime \prime}(p=0,001)$. Isto nos mostrou que quanto maior é a pontuação do teste de Tinetti menor é tempo no teste Timed "Up \& Go".

Por fim encontrou-se uma correlação positiva entre o Tinetti equilíbrio e o Berg ( $r=0,571 ; p=0.000)$ e para o grupo controle $(r=0,826$; $p=0,000)$. Também houve uma correlação positiva entre o Tinetti total com o BERG entre os grupos experimentais $(r=0,599 ; p=0,000)$ e o controle $(r=0,653 ; p=0,001)$.

\section{DISCUSSÃO}

Várias revisões e estudos controlados atribuem a prática regular de atividade física, mesmo quando iniciada após os 65 anos, como algo que favorece uma maior longevidade, a redução das taxas gerais de mortalidade, e do número de medicamentos prescritos, a prevenção do declínio cognitivo, a manutenção de status funcional, a redução da freqüência de quedas e a incidência de fraturas, além dos benefícios psicológicos como a melhora da auto-estima ${ }^{(11)}$.

Um recente estudo realizado por Ribeiro e Pereira ${ }^{(12)}$ conseguiu demonstrar que a Escala de Equilíbrio de Berg é a mais acurada para detectar alterações no equilíbrio nos idosos saudáveis. Os idosos dos grupos experimentais e controle não apresentaram diferenças significativas nos resultados da Escala de Equilíbrio de Berg. Os dados desse estudo mostraram que não houve diferenças significativas entre as médias na Escala de Equilíbrio de Berg. No presente estudo também não se encontrou diferença estatisticamente significativa para a Escala de Equilíbrio de Berg entre os grupos.

A diminuição no tempo do Time Up \& Go apresentada no presente estudo se fez importante, pois este teste tem uma grande relação com o equilíbrio, a velocidade da marcha e a capacidade funcional|(13), ou seja, tudo o que está relacionado diretamente com a propensão para as quedas. Portanto, o tempo gasto para a realização do teste está diretamente associado ao nível da mobilidade funcional. Tempos reduzidos na realização do teste indicam idosos independentes quanto à mobilidade. Já os idosos que o realizam em um tempo superior aos 20 s tendem a ser mais dependentes nas suas tarefas diárias ${ }^{(13,14)}$.

Guimarães et al. ${ }^{(15)}$ utilizaram o teste "Time Up \& Go" para avaliar o nível de mobilidade funcional entre os idosos sedentários e os ativos, concluindo que os idosos que praticavam atividades físicas levaram menor tempo para realização do teste quando comparados com os sedentários, algo que também foi encontrado neste estudo.

O presente estudo demonstrou que um programa de exercícios de força progressiva em homens idosos pode melhorar as suas capacidades funcionais como a do equilíbrio, da coordenação e da agilidade, além de minimizar os fatores de risco de quedas. Estes resultados vão de encontro aos apresentados por Rubenstein et al. ${ }^{(16)}$, bem como os de outros autores, os quais relataram uma melhora da resistência muscular e da funcionalidade nos homens idosos após um programa simples de exercícios de resistência progressiva, de caminhada e de treino de equilíbrio ${ }^{(17-20)}$.

Todos estes resultados, ora mencionados, são finalizações clinicas importantes para uma população com deficiências motoras, porque mesmo que as melhorias da resistência, da força e da mobilidade sejam mínimas, elas têm impactos para que o indivíduo continue sem depender de terceiros. Uma outra pesquisa anterior mostrou que o exercício tem um impacto positivo no desempenho físico e na independência das pessoas mais velhas com problemas funcionais em relação às outras.

Rubenstein et al. ${ }^{(16)}$ em seu estudo, demonstraram que um programa simples com exercícios de resistência, com treinamentos progressivos com contrapesos e o caminhar, pode melhorar a resistência do músculo e a mobilidade funcional nos homens idosos. Além disso, este estudo fornece uma evidência nova no relacionamento complexo entre a atividade física e as pessoas propensas a sofrerem quedas, os participantes do exercício aumentaram significativamente a sua atividade física, embora tenha sofrido poucas quedas em virtude da sua atividade. O benefício físico a curto prazo mais notável associado a este programa parece ser uma melhoria total das resistências física e funcional.

Rubenstein et al. ${ }^{(16)}$ mostraram nos seus resultados que houve uma melhora significativa na pontuação do teste Tinetti marcha para o grupo exercício comparado com o controle. O que podemos observar neste presente estudo é que, o grupo experimental também mostrou uma melhor pontuação no teste de Tinetti marcha quando comparado com o controle.

Embora programas de exercícios possam melhorar a força muscular, a marcha e o equilíbrio entre as pessoas idosas, há evidências que o exercício diminuiu o número de quedas (21).

É importante ressaltar que o envelhecimento populacional e o aumento da expectativa de vida demandam ações preventivas, as quais possam controlar os fatores de risco das quedas e promovam medidas que visem a participação dessa população à prática de atividade física.

A atividade física é uma mobilidade terapêutica que melhora a mobilidade física e a estabilidade postural, as quais estão diretamente relacionadas com a diminuição de quedas(22). Muitos estudos têm examinado os efeitos do exercício físico sobre a estabilidade postural. A participação em programas de exercícios de intensidade leve tem-se demonstrado útil para a redução significativa do número de quedas, quando se compara os grupos controle que não realizam exercícios com os que os realizam ${ }^{(13,23)}$. 
O presente estudo apresentou algumas limitações como a da falta da avaliação pré e pós-intervenção e a falta do cálculo do tamanho da amostra; a da falta da avaliação da força muscular pela dinamometria isocinética; e a da aplicação do treinamento neuromuscular para se comparar com o treinamento de resistência.

Por isso, futuras pesquisas, englobando outras variáveis e com protocolos reprodutíveis, devem ser realizadas a fim de se verificar qual seria o melhor tipo de exercício (força, resistência, neuromuscular, aeróbio, anaeróbio, intervalado ou combinado) e o mais efetivo para a melhora do desempenho funcional e, conseqüentemente, na prevenção de quedas nos idosos.

\section{CONCLUSÕES}

De acordo com os dados apresentados na comparação entre os grupos, podemos concluir que o programa de treinamento de resistência a 80\% de 1 RM, durante 24 semanas, mostrou-se favorável na melhora do equilíbrio, da coordenação e da agilidade nos idosos.

\section{AGRADECIMENTOS}

Os Autores gostariam de agradecer o apoio da Fundação de Amparo à Pesquisa do Estado de São Paulo (FAPESP), da Associação Fundo de Incentivo à Psicofarmacologia (AFIP), do Centro de Estudos em Psicobiologia e Exercício (CEPE), ao CEPID/SONO/FAPESP, CNPQ, FADA/ UNIFESP e ao CENESP/UNIFESP.

Todos os autores declararam não haver qualquer potencial conflito de interesses referente a este artigo.

\section{REFERÊNCIAS BIBLIOGRÁFICAS}

1. Overstall PW. The use of balance training in elderly people with falls, Reviews in Clinical Gerontology 2003; 13: 153-61.

2. Pereira SEM, Buksman S, Perracini M, Py L, Barreto KML, Leite VMM. Projeto Diretrizes: Quedas em Idosos. Sociedade Brasileira de Geriatria e Gerontologia. 2001.

3. Miyamoto ST, Lombardi J, Berg KO, Ramos LR, Natour J. Brazilian version of the Berg balance scale Braz J Med Biol Res 2004; 37: 1411-21.

4. Hayes KW, Johnson ME. Berg balance scale. American College of Rheumatology 2003; 4: 28-30.

5. Berg K, Wood-Dauphinée S, Williams JI. Measuring balance in the elderly: preliminary development of an instrument. Physiotherapy Canada 1989; 41: 304-11.

6. Berg K, Maki B, Williams J. Clinical and laboratory measures of postural balance in an elderly population. Arch Phys Med Rehabil 1992; 73: 1073-80.

7. Shumway-Cook A, Woollacott MH. Uma estrutura conceitual na prática clínica. In: Controle Motor: Teoria e Aplicações Práticas. 2a ed., Manole, São Paulo, 2003.

8. Kenneth A, Behm D. O Impacto do Treino de Resistência à Instabilidade no Equilíbrio e Estabilidade. Sports Med 2005; 35: 43-53

9. Lajoie Y, Gallagher SP. Predicting falls within the elderly community: comparison of postural sway, reaction time, the Berg balance scale and the Activities-specific Balance Confidence (ABC) Scale for comparing fallers and non-fallers, Archives of Gerontology and Geriatrics 2004: 38: 11-26.

10. Shumway-Cook A, Baldwin M, Polissar NL, Gruber W. Predicting the probability for falls in communitydwelling older adults. Physical Therapy 1997; 77: 812-9.

11. Elward K, Larson EB. Benefits of exercise for older adults. A review of existing evidence and current recommendations for the general population. Review. Clin Geriatr Med 1992; 8: 35-50.

12. Ribeiro ASB, Pereira JS. Melhora do equilíbrio e redução da possibilidade de quedas em idosas após os exercícios de Cawthorne e Cooksey. Rev Bras Otorrinolaringol 2005; 7: 38-46.
13. Podsiadlo D, Richardson S. The Timed Up \& Go: A test of basic functional mobility for frail elderly persons. J Am Geriatr Soc 1991; 39: 142-8.

14. Worsfold C, Simpson JM. Sttandardisation of a Three-metre Walking Test fot Elderly People. Physiotherapy 2001; 87: 125-32.

15. Guimarães LHCT, Galdino DCA, Martins FLM, Vitorino DFM, Pereira KL, Carvalho EM. Comparação da propensão de quedas entre idosos que praticam atividade física e idosa sedentários. Revista Neurociências 2004; 12. Disponível em http://www.unifesp.br/dneuro/neurociencias/vol12_2/quedas.htm

16. Rubenstein LZ, Josephson KR, Trueblood PR et al. Effect of a Group Exercise Program on Strength, Mobility, and Falls Among Fall-Prone Elderly Men. J Gerontol Med Sci 2000; 55: 317-21.

17. Buchner DM, Cress ME, Lateur BJ, Esselman PC, Margherita AJ, Price R et al. The effect of strength and endurance training on gait, balance, fall risk, and health services in community-living older adults. J Gerontol 1997; 2: 218-24.

18. Tinetti ME, Baker DI, McAvay G, Claus EB, Garrett P, Gottschalk M, et al. A multifactorial intervention to reduce the risk of falling among elderly people living in the community. N Engl J Med 1994; 331: $821-7$.

19. Gillespie LD, Gillespie WJ, Cumming R, Lamb SE, Rowe BH. Interventions to reduce the incidence of falling in the elderly (Cochrane Review). In: The Cochrane Library, Issue 3, 1998. Oxford: Update Software.

20. Anderson K, Behm DG. The Impact of Instability Resistance Training on Balance and Stability. Sports Med 2005; 35: 43-53.

21. Morgan RO, Virnig BA, Duque M, Abdel-Moy E, De Vito CA. Low-Intensity Exercise and Reduction of the Risk for Falls Among At-Risk Elders. Journal of Gerontology 2004; 59: 1062-7.

22. Thomas, SG. Programas de Exercícios e Atividades. In: Pickles B et al. Fisiologia na 3a Idade. 2.ed. São Paulo: Santos, 2000: 158-67.

23. Mazzeo RS, Cavanagh P, Evans WJ. Exercício e atividade física para pessoas idosas. Revista Brasileira de Atividade Física \& Saúde, 1998. 